\title{
ORIGINAL ARTICLE Preclinical targeting of aggressive T-cell malignancies using anti-CD5 chimeric antigen receptor
}

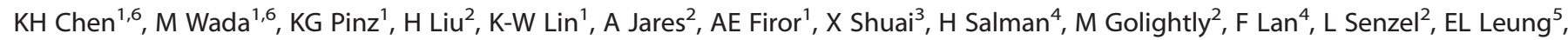 \\ $X$ Jiang ${ }^{1}$ and $Y \mathrm{Ma}^{1,2,5}$
}

The outlook for T-cell malignancies remain poor due to the lack of effective therapeutic options. Chimeric antigen receptor (CAR) immunotherapy has recently shown promise in clinical trials for B-cell malignancies, however, designing CARs for T-cell based disease remain a challenge due to the shared surface antigen pool between normal and malignant T-cells. Normal T-cells express CD5 but NK (natural killer) cells do not, positioning NK cells as attractive cytotoxicity cells for CD5CAR design. Additionally, CD5 is highly expressed in T-cell acute lymphoblastic leukemia (T-ALL) and peripheral T-cell lymphomas (PTCLs). Here, we report a robust anti-CD5 CAR (CD5CAR) transduced into a human NK cell line NK-92 that can undergo stable expansion ex vivo. We found that CD5CAR NK-92 cells possessed consistent, specific, and potent anti-tumor activity against a variety of T-cell leukemia and lymphoma cell lines as well as primary tumor cells. Furthermore, we were able to demonstrate significant inhibition and control of disease progression in xenograft mouse models of T-ALL. The data suggest that CAR redirected targeting for T-cell malignancies using NK cells may be a viable method for new and complementary therapeutic approaches that could improve the current outcome for patients.

Leukemia (2017) 31, 2151-2160; doi:10.1038/leu.2017.8

\section{INTRODUCTION}

The prognosis for patients with T-cell acute lymphoblastic leukemia (T-ALL) and peripheral T-cell lymphomas (PTCLs) remain poor, with no potential curative option beyond chemotherapy and its large trade-offs in potential short and long-term toxicities. ${ }^{1}$ In recent years, CAR therapy has shown promise as a powerful new adoptive immunotherapy technique for a number of solid and hematological cancers, most notably B-cell lymphoma. ${ }^{2-4}$ CAR therapy utilizes modified patient immune cells, traditionally $T$ and more recently NK cells, ${ }^{5,6}$ to target and eliminate malignancies in a major histocompatibility complex-independent manner. Strategies targeting T-cell malignancies using CAR-mediated therapy against T-cell antigens have been limited due to concern over the potentially higher immunodeficiency impact of T-cell depletion vs B-cell aplasia for current CAR-directed anti-CD19 clinical trials. ${ }^{3}$ In addition, directing CAR-modified T-cells against shared T-cell antigens present on malignant cells could result in self-targeting and compromise of therapeutic ability. We hypothesized that T-cell self-targeting could potentially be mitigated by using NK cells instead.

NK cells are $\mathrm{CD}^{-}$and $\mathrm{CD}^{-}$large granular lymphocytes constituting an important part of the innate immune system. Their use as anti-cancer effector cells have been well documented in targeting a variety of malignancies ${ }^{7-10}$ and, importantly, possess different cytotoxic and persistency mechanisms that may be utilized as an alternative or complementary type of therapy from T-cells. ${ }^{11-14}$ One differentiating factor is that the short NK cells lifespans relative to T-cells prime NK cells for transient immunotherapy ${ }^{11}$ and the lack of shared antigens would preclude self-targeting and compromise of immunologic function. CARmodified NK cells are expected to be exhausted shortly after tumorlysis, with a turnover time between a week to 2 weeks. ${ }^{11}$ This transient effect may preclude the need for an inducible safety switch. ${ }^{2,15,16}$ In addition, NK cells have been seen to mediate antitumor effects with little risk of graft-versus-host disease and have been validated in CAR applications ${ }^{11,13}$ as well as efficacy in a number of clinical trials targeting solid tumor and hematological malignancies. ${ }^{9,14,17-21}$

CD5 is not expressed in hematopoietic stem cells and other non-hematopoietic cells but is a characteristic surface marker expressed in a majority of T-cell malignancies including T-ALL and T-lymphoma in addition to some B-cell lymphomas. ${ }^{22,23}$ The antiCD5 SCFv domain in our CD5CAR design is derived from an established CD5-monoclonal antibody that has been used in previous clinical trials without irreversible off-target effects. ${ }^{24-27}$

In this proof-of-principle work for NK cells, we hypothesized that a CD5-directed NK cell therapy can be used as an alternative approach to target T-cell malignancies. In our studies, we show that CD5CAR NK-92 cells specifically target and eliminate both $\mathrm{CD}^{+}$tumor cell lines and $\mathrm{CD}^{+}$primary tumor cells in vitro. We also demonstrate potent tumor-directed cytotoxicity in two mouse xenograft models with significantly improved survival outlooks for CAR treated mice.

\footnotetext{
${ }^{1}$ iCell Gene Therapeutics LLC, Research \& Development Division, Long Island High Technology Incubator, Stony Brook, NY, USA; ${ }^{2}$ Department of Pathology, Stony Brook Medicine, Stony Brook, NY, USA; ${ }^{3}$ Department of Hematology, West China Hospital, Sichuan University, Chengdu, Sichuan, China; ${ }^{4}$ Department of Internal Medicine, Stony Brook Medicine, Stony Brook University Medical Center, Stony Brook, NY, USA and ${ }^{5}$ Faculty of Chinese Medicine, State Key Laboratory of Quality Research in Chinese Medicine, Macau University of Science and Technology, Macau, China. Correspondence: Dr X Jiang or Dr Y Ma, iCell Gene Therapeutics LLC, Research \& Development Division, Long Island High Technology Incubator, 25 Health Sciences Drive, Suite 118, Stony Brook, NY 11790, USA.

E-mail: sue.jiang@icellgene.com or yupo.ma@icellgene.com

${ }^{6}$ These authors contributed equally to this work.

Received 21 September 2016; revised 16 November 2016; accepted 12 December 2016; accepted article preview online 12 January 2017 ; advance online publication, 10 February 2017
} 


\section{MATERIALS AND METHODS}

Primary tumor cells and cell lines

Human primary tumor samples were obtained from residual bonemarrow aspirate samples after final diagnosis was made according to compliance protocols. KARPAS 299, CCRF-CEM, Jurkat, MOLT-4, JeKo and NK-92 cell lines were obtained from ATCC (Manassas, VA). NK-92 cells were cultured in NK-92 cell media (defined as alpha-MEM without ribonucleosides and deoxyribonucleosides with $2 \mathrm{~mm}$ L-glutamine, $1.5 \mathrm{~g} / \mathrm{l}$ sodium bicarbonate, $12.5 \%$ heat-inactivated horse serum, $12.5 \%$ heat-inactivated FBS, $1 \times$ Pen/Strep, $0.2 \%$ inositol, $0.02 \%$ folic acid and 50 um beta-mercaptoethanol) supplemented with IL-2 $(300 \mathrm{IU} / \mathrm{ml})$ and fresh media every 2 days to a maintenance cell density of $0.3-1 \times 10^{6}$ cells $/ \mathrm{ml}$. NK-92 cells were then maintained for up to 3 months for in vitro and in vivo experiments. KARPAS 299, CCRF-CEM and Jurkat cell lines were cultured in RPMI, 10\% FBS, $1 \times$ Pen/Strep (Gibco, Waltham, MA, USA).

Co-culture assays and specific cytotoxicity assays

CD5CAR and vector control NK-92 cells were incubated with CD5 expressing T-ALL cell lines: Jurkat $(n=4)$, CCRF-CEM $(n=4)$, MOLT-4 $(n=2)$, in addition to primary patient cells: $\mathrm{CD}^{+}$umbilical cord blood (UCB; $n=4)$ or peripheral blood ( $\mathrm{PB} ; n=2)$ derived T-cells, and CD5 expressing primary human T-lymphoma cells: SPT-1 (adult Sezary syndrome; $n=2$ ) and PT4 (unclassified PTCL; $n=4$ ). In addition, CD5CAR NK-92 cells were co-cultured with T-ALL primary leukemia cells: T-ALL 1 (majority $\mathrm{CD} 4^{+} \mathrm{CD}^{-}$tumor burden, $n=2$ ) and T-ALL 2 (majority $\mathrm{CD}^{+}$ $\mathrm{CD}^{+}$tumor burden and cytoplasmic $\left.\mathrm{CD}^{+}, n=4\right)$. CD5CAR NK-92 cells were also used against $C D 5$ expressing mantle cell lymphoma (MCL) cell line JeKo $(n=2)$ and primary MCL sample L3-G $(n=2)$. For specific population lysis assays, PT4, T-ALL 2, UCB/PB T-cells and T-ALL 1 were used. As a negative control, CD5CAR and vector NK-92 cells were incubated with CD5-negative non-Hodgkin's Lymphoma cell line KARPAS $299(n=2)$

Specific cytotoxicity assays were carried as described previously ${ }^{10}$ with Jurkat, and CCRF-CEM cell lines.

Analysis of anti-leukemic activity was performed as described previously. ${ }^{10}$ For a list of antibodies and reagents used, see Supplementary Table S1.

Additional methods and materials are listed in Supplementary Methods consisting of CD5CAR design, specific conditions and mouse model construction and analysis.

\section{RESULTS}

Characterization of CD5CAR construct and expansion of CAR NK-92 cells

The CD5CAR design is comprised of anti-CD5 scFv, a CD8-derived hinge $(\mathrm{H})$ and transmembrane region, and $\mathrm{CD} 28$ and 4-1BB intracellular signaling domains in tandem with the CD3 zeta signaling domain (Figure 1a). Western blot analysis was performed and immunoblotting with anti-CD3 zeta monoclonal antibody produced bands of predicted size $(57.86 \mathrm{kDa})$ for the CD5CAR-CD3 zeta fusion protein (Figure 1b). There were no bands observed for the vector control lane as expected.

After transduction, the efficiency of the CD5CAR was determined by flow cytometry analysis to be $26.5 \%$ CD5CAR (Figure 1c). To enrich for CD5CAR ${ }^{+}$NK cells, the NK cells that expressed the construct most avidly were harvested by fluorescence activated cell sorting. Following sorting, CD5CAR ${ }^{\text {high }}$ NK-92 cells were expanded in vitro for 3-4 months with stable expression.

CD5CAR NK-92 cells eliminate T-ALL and lymphoma cell line cells in vitro

We evaluated the anti-tumor activity of CD5CAR NK-92 cells using $\mathrm{CD}^{+}{ }^{\mathrm{T}}$-ALL cell lines (Supplementary Figure 1). A CD5-negative anaplastic large cell lymphoma cell line, KARPAS was used as a negative control (Supplementary Figure 1). CD5CAR NK-92 cells were tested via 6 hour or overnight co-cultures in vitro using CCRF-CEM $(n=4)$, MOLT-4 $(n=2)$, and Jurkat $(n=4)$ cell lines with effector:target (E:T) cell ratios of 2:1 and 5:1. Compared to vector control NK-92 cells, we observed that CD5CAR NK-92 cells consistently and robustly eliminated all $\mathrm{CD}^{+}$populations (Figure 2c). At an E:T ratio of 2:1, we find that CD5CAR NK-92 cells already demonstrate significant cytotoxicity ( 90\%) (Figure 2a). With an E:T increase to $5: 1$, virtually $100 \%$ lysis was observed for all three T-ALL cell lines (Figure 2b). No lysis was observed of the $\mathrm{CD} 4^{+} \mathrm{CD}^{-}$KARPAS cell line for either E:T ratio $(n=2)$, highlighting targeting specificity to CD5 expressing cells (Figures $2 \mathrm{a}$ and $\mathrm{b}$, Supplementary Figure 1).

It is possible that NK cell expansion during target antigen binding can confound cytotoxicity measurements. Thus we employed two measures to verify the robustness of the

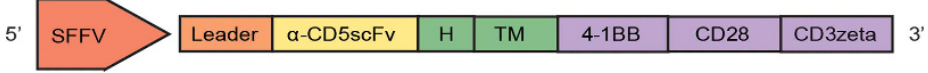

b

\section{Western Blot C CD5-3G NK-92 CAR Surface Expression}
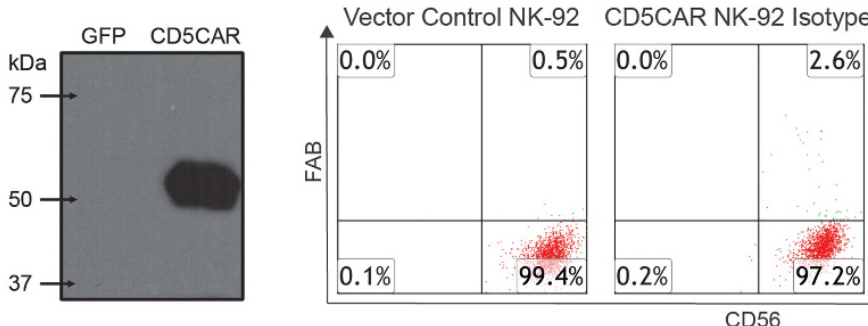

CD5CAR NK-92

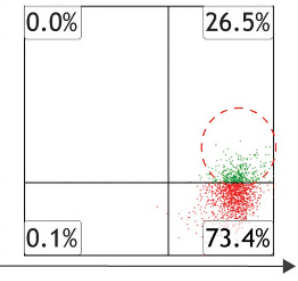

Figure 1. Organization of the CD5CAR NK-92 construct and its expression. (a) Schematic representation of the CD5CAR NK-92 lentiviral vector. The CD5CAR NK-92 construct is a modularized signaling domain containing: a leader sequence, an anti-CD5scFv, a hinge domain (H), a transmembrane domain, two co-stimulatory domains (CD28 and 4-1BB) and the intracellular signaling domain CD3 zeta. (b) Western Blot analysis of CD5CAR. GFP (lane 1) and CD5CAR NK-92 (lane 2) were used to transduced HEK-293FT cells and subsequently probed with mouse anti-human CD3 zeta antibody $48 \mathrm{~h}$ post transduction for Western blot analysis. The expected weight of the CD5CAR NK-92 construct is $57.86 \mathrm{kDa}$ by sequence analysis data (not shown). (c) Flow cytometry analysis of CD5CAR NK-92 expression on NK-92 cell surface (right) vs vector control NK-92 cells (left) and CD5CAR isotype control (middle). The population in green delineates transduced NK-92 cell population after fluorescence activated cell sorting. Gating was based off the vector and isotype controls. 
a

CD5CAR NK-92 + CD5 ${ }^{+}$Malignant Cell Line Co-Cultures $~ E: T=2: 1$

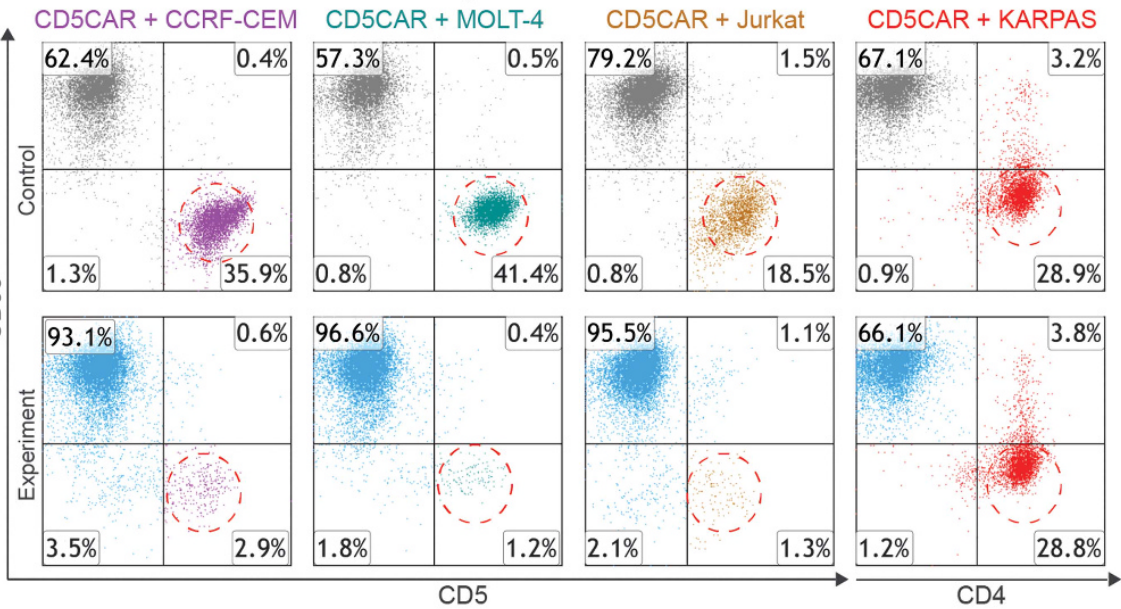

b

CD5CAR NK-92 + CD5 ${ }^{+}$Malignant Cell Line Co-Cultures $\sim \mathrm{E}: \mathrm{T}=\mathbf{5 : 1}$
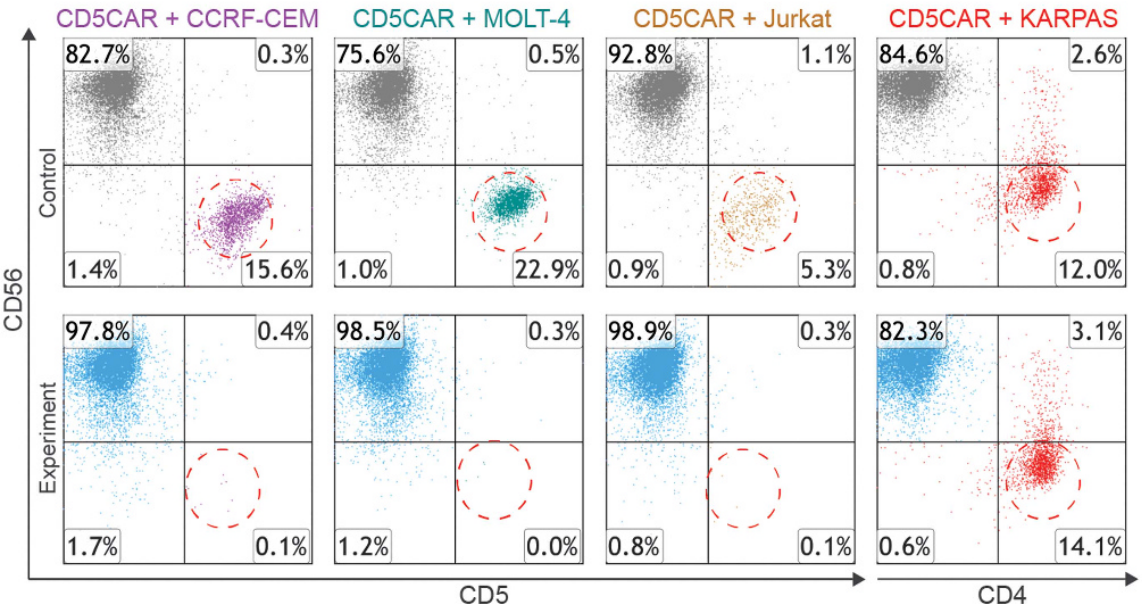

C

Malignant Cell Line Co-Cultures at an $E: T$ of $2: 1$ and $5: 1$

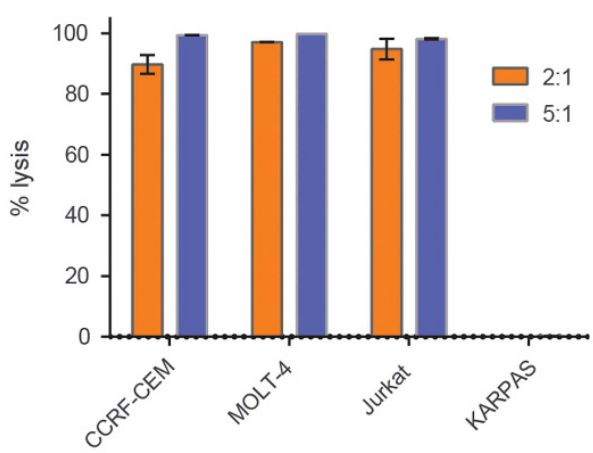

d

Absolute cell counts of E:T 5:1 co-cultures CD5CAR + CCRF-CEM CD5CAR + MOLT-4 CD5CAR + Jurkat

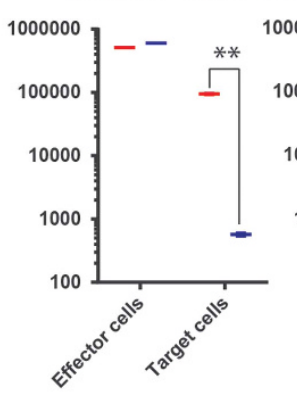

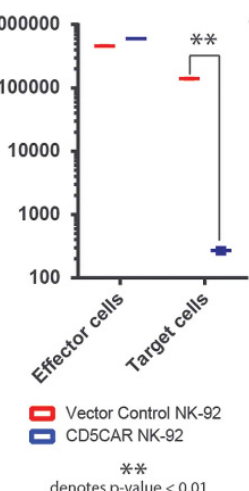

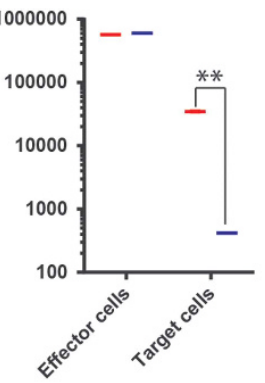

Figure 2. CD5CAR NK-92 cells eliminate CD5 expressing T-ALL cell lines in vitro. (a) Co-cultures at an E:T ratio of 2:1 using CD5CAR NK-92 cells against malignant cell lines using CD56 and CD5 to delineate the NK cell and target cell populations respectively. Target cell survival in experiment is expressed relative to target cell survival in vector control NK treatment. Each bar graph represents the average statistics for duplicate samples with $n=4$. T-ALL cell line Jurkat was co-cultured for $6 \mathrm{~h}$ while all other cell types were cultured overnight. CD4 was used to identify negative control NHL cell line KARPAS cells. Populations encircled to show target cells of interest. (b) Co-cultures with CD5CAR NK-92 cells performed at an E:T ratio of 5:1 with the same experimental conditions. (c) Summary of CD5CAR NK-92 cytotoxicity against T-ALL and T-lymphoma cells lines. (d) Absolute cell counts of CCRF-CEM, MOLT-4 and Jurkat co-cultures of both effector and target cells. Control treatments are delineated in red while CD5CAR treatments are in blue. 
co-culture assays. Absolute cell counts were performed to demonstrate significant depletion of total target cell populations. Second, specific cytotoxicity assays were performed as described previously ${ }^{10}$ where $\%$ specific lysis was measured by comparing the survival of $\mathrm{CD}^{+}$target cells relative to the survival of negative control cells in the same treatment condition. ${ }^{28}$ Although NK-92

a CD5CAR NK-92 + CD5+T-ALL/Leukemic Patient Sample Co-Cultures

$$
\mathrm{E}: \mathrm{T}=2: 1
$$
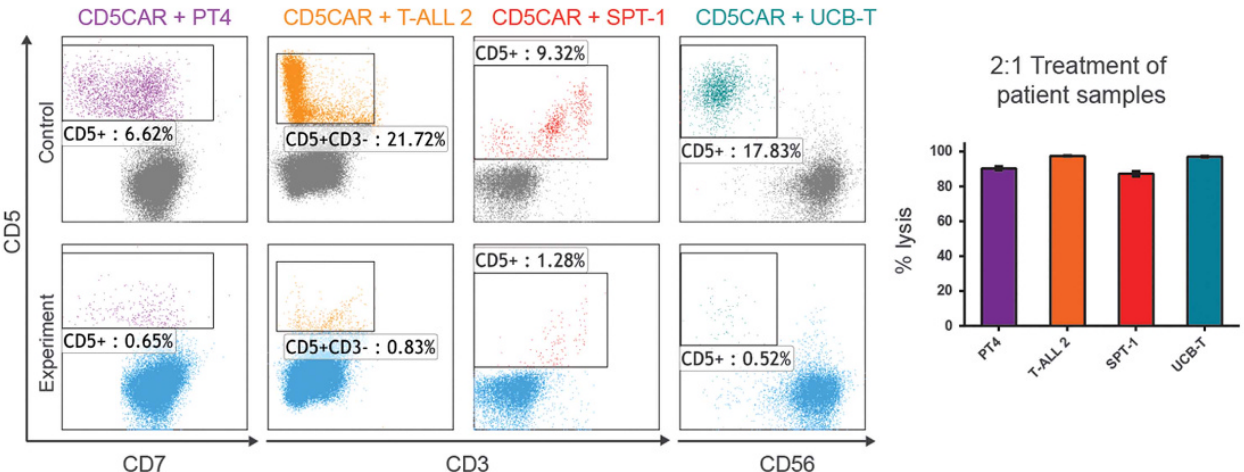

b

CD5CAR NK-92 + CD5 ${ }^{+}$T-ALL/Leukemic Patient Sample Co-Cultures $\mathrm{E}: \mathrm{T}=5: 1$

CD5CAR + PT4

CD5CAR + T-ALL 2 CD5CAR + SPT-1 CD5CAR + UCB-T
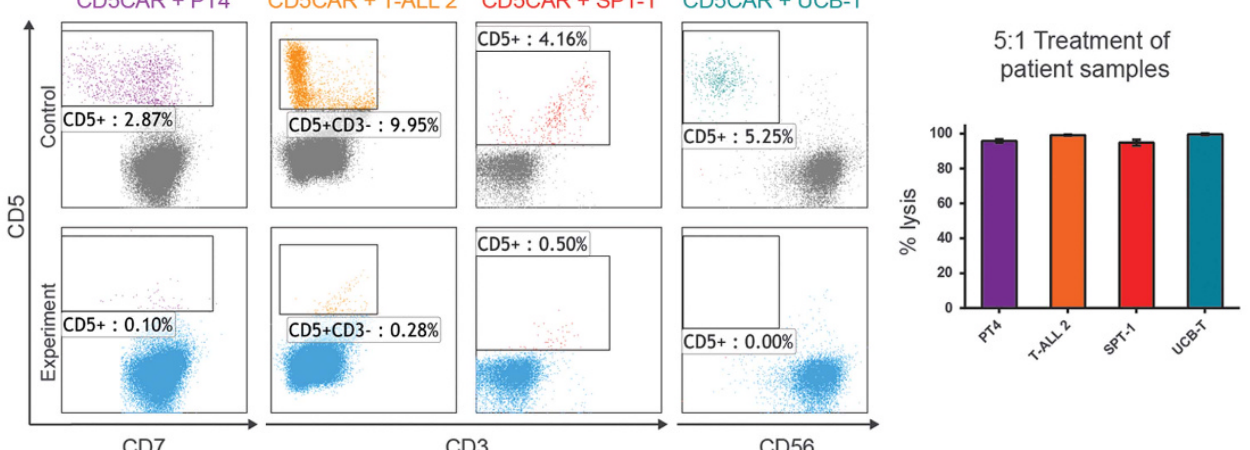

C

CD5CAR NK-92 + CD5 ${ }^{+}$MCL Co-Cultures
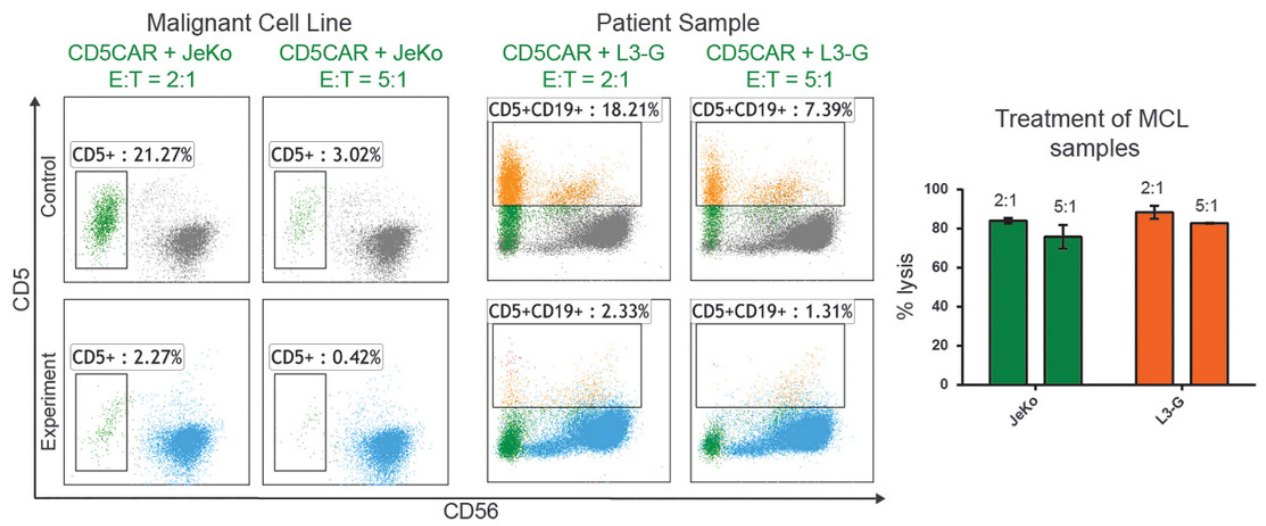

d

Absolute cell counts of patient E:T 5:1 co-cultures

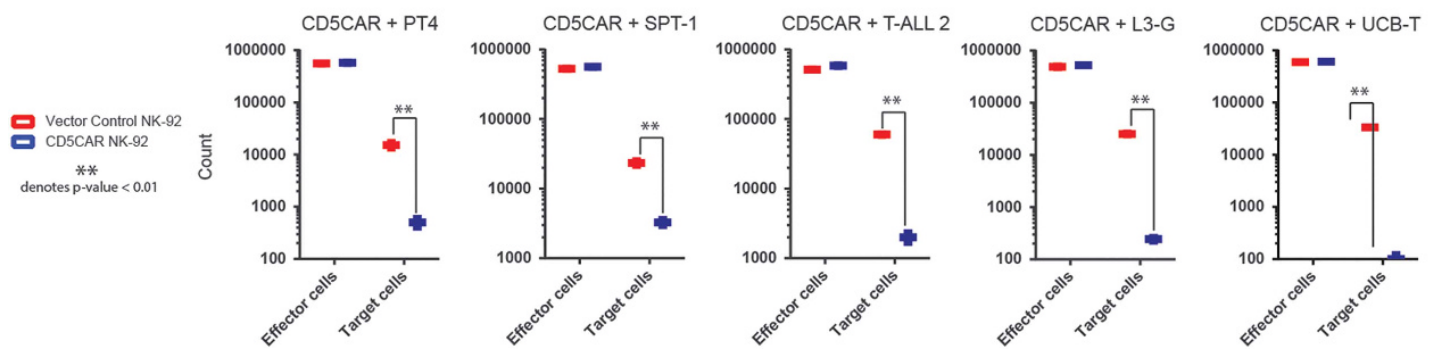


cells have insufficient time during a typical co-culture experiment $(<24 \mathrm{~h})$ to expand $(\sim 72 \mathrm{~h})$, absolute cell counts of both effector and target cell populations reveal highly statistically significant differences in target cell populations as a result of tumorlysis (Figure 2d). Furthermore, the specific cytotoxicity assays against Jurkat and CCRF-CEM cells show consistent and comparable cytotoxicity numbers with the co-cultures performed above (Supplementary Figure 2). The specific cytotoxicity assays also show that control NK-92 cells exhibit intrinsic cell-lytic ability against Jurkat cells, albeit at an E:T ratio of 5:1 only, with negligible effect at lower doses (Supplementary Figure 2). This might be due to the activation of the Fas-fas ligand pathway by NK-92 cells against Fas ${ }^{+}$Jurkat cells.

CD5CAR NK-92 cells recognize and lyse aggressive $C D 5^{+}$primary T-ALL leukemic cells

We then tested the efficiency of CD5CAR NK-92 cells in recognizing and killing primary tumor cells. Co-culture experiments were conducted using patient samples T-ALL $1(n=2)$ and T-ALL $2(n=4)$ from leukemia patients unresponsive to standard chemotherapy. The phenotype of T-ALL 1 includes a small subset of T-ALL cells positive for CD5 ( 14\%) consisting of leukemic $\mathrm{CD} 4^{+} \mathrm{CD}^{+}$cells as well as a small population of 'phenotypically normal' $\mathrm{CD} 34^{-} \mathrm{CD}^{+}$T-cells (Supplementary Figure 3F). Target populations were gated and quantified with flow cytometry using cell Cytotracker dye (CMTMR) to label T-ALL cells. We find that CD5CAR NK-92 cells target and are cytotoxic against the leukemic $\mathrm{CD}_{3} 4^{+} \mathrm{CD}^{+}$tumor cells as well as the normal T-population (Supplementary Figure 4). In contrast, CD5CAR NK-92 cells showed no lytic activity against the majority $\mathrm{CD}^{-}$cell population, implying specific and directed activity against target antigen epitopes.

The phenotype of the T-ALL 2 sample comprises of an $\sim 88 \%$ leukemic population that was potently lysed by CD5CAR NK-92 cells at high efficiency under an E:T ratio of 2:1, and approached $100 \%$ lysis when increased to a ratio of $5: 1$ (Supplementary Figure 3C, Figures $3 \mathrm{a}$ and $\mathrm{b}$ ). In addition, we conducted absolute cell counts that show T-ALL 2 target cells were significantly depleted during co-culture (Figure 3d).

CD5CAR NK-92 cells target and eliminate primary PTCL and Sézary tumor cells

Next, we evaluated the ability of CD5CAR NK-92 cells to kill primary T-lymphoma cells. An unclassified PTCL PT4 $(n=4)$ and chemotherapy resistant Sézary syndrome SPT-1 $(n=2)$ expressing CD5 primary sample were used as target populations (Supplementary Figure 3D, 3E). The phenotype of PT4 consisted of $\mathrm{CD}^{+} \mathrm{CD}^{-}$malignant cells (Supplementary Figure $3 \mathrm{E}$ ) which were lysed at high efficiency approaching total ablation (Figures $3 a$ and b). The $\mathrm{CD}^{+}$Sézary population was also highly lysed by CD5CAR NK-92 cells, with substantial cell ablation at all E:T ratios (Figures 3a and b). Both PT4 and SPT-1 target cell populations were significantly depleted as demonstrated by absolute cell counts (Figure 3d).
CD5CAR NK-92 cells deplete CD5 ${ }^{+}$normal human UCB T cells

To assess the cytotoxicity of CD5CAR NK-92 cells against normal T-cells, we co-cultured CD5CAR NK-92 cells against UCB-derived T-cells $(n=6)$. We find that UCB T-cells are significantly depleted at all E:T ratios (Figures $3 a$ and $b$ ).

\section{CD5CAR NK-92 cells lyse CD5 ${ }^{+} \mathrm{MCL}$ cells}

Additional co-culture studies were conducted to assess CD5CAR activity against difficult to treat $\mathrm{CD}^{+}$mantle cell lymphoma with very few curative options. Cultures were conducted using the cell line JeKo $(n=2)$ established from PB mononuclear cells of a large cell variant of MCL as well primary lymphoma sample L3-G $(n=2)$ (Supplementary Figure 2). In co-culture studies at an E:T of 2:1, CD5CAR NK-92 cells lysed $\sim 80 \%$ of Jeko cells (Figure 3c). Similarly, flow cytometry analysis shows depletion of the broad $\mathrm{CD}^{+}$L3-G population, with $>95 \%$ lysis efficiency at lower E:T ratios (Figure $3 \mathrm{c}$ ). Absolute cell counts confirmed the highly significant depletion of target cell populations (Figure 3d).

Efficient targeting and lysis of $\mathrm{CD}^{+}$cells within heterogeneous primary tumor cell populations

To further investigate the specificity of CD5CAR NK-92 cells in targeting $\mathrm{CD}^{+}$populations and potential off-target effects, we analyzed co-culture assays with T-ALL 2 and L3-G utilizing multiple markers to determine the effect of CD5CAR NK-92 treatment on each heterogeneous primary patient sample.

Flow cytometry labeling with CD34, CD3 and CD5 revealed that T-ALL 2 consists of two distinct leukemic cell populations: a majority $\mathrm{CD}^{+} \mathrm{CD}^{-} \mathrm{CD}^{-} 4^{-}$and a minority $\mathrm{CD}^{+} \mathrm{CD}^{-} \mathrm{CD} 34^{+}$ subset (Supplementary Figure 3C). In co-cultures with CD5CAR NK-92, we observed that the majority $\mathrm{CD}^{+} \mathrm{CD}^{-} \mathrm{CD}^{-} 4^{-}$leukemic population was virtually eliminated by CD5CAR treatment $(>98 \%$ cytotoxicity; Figure $4 a$ ). The minority $\mathrm{CD}^{+} \mathrm{CD}^{-} \mathrm{CD}^{-} 4^{+}$population phenotypically exhibited a lower average level of CD5 expression. However, CD5CAR NK-92 cells still lysed this population at $>80 \%$ efficiency (Figure 4a). Of note was the third $\mathrm{CD}^{-} \mathrm{CD}^{-} \mathrm{CD}^{-} 4^{+}$ population that remained unaffected by CD5CAR treatment.

Flow cytometry analysis with CD19 and CD5 separated the L3-G population into three distinct groups: a majority B-cell lymphoma $\mathrm{CD}^{+} \mathrm{CD} 19^{+}$population, a minority $\mathrm{CD}^{-} \mathrm{CD} 19^{+}$population and $\mathrm{a}$ small $\mathrm{CD}^{+} \mathrm{CD}^{-} 9^{-}$T-cell population (Supplementary Figure 3B). The $\mathrm{CD}^{+} \mathrm{CD} 19^{+}$lymphoma population was lysed by CD5CAR NK-92 at $>90 \%$ efficiency at an E:T ratio of 2:1, with a slight decrease in lysis efficiency at higher (standard) E:T ratios that may suggest a saturation of lytic activity. The $\mathrm{CD}^{+} \mathrm{CD}^{+} 9^{-}$T-cell population was targeted as strongly or more so, with a complete depletion of target cells at an E:T of 5:1 (Figure 4b). In contrast the $\mathrm{CD}^{-} \mathrm{CD} 19^{+}$non-target population showed no significant effect (Figure 4b). These results suggest that the CD5CAR NK-92 cells lyse $\mathrm{CD}^{+}$target cell populations with high specificity and potency.

CD5CAR NK-92 cells demonstrate dose-dependent tumor-lytic efficiencies for multiple $\mathrm{CD}^{+}$populations in vitro

We hypothesized that NK cell therapy may be posited as a transient therapy whereupon effector cells will be exhausted and

Figure 3. CD5CAR NK-92 cells specifically target and eliminate aggressive CD5 ${ }^{+}$primary T-ALL and T-lymphoma cells. (a) At a 2:1 E:T ratio, the CD5CAR NK-92 cells specifically lyse the $\mathrm{CD}^{+}$populations of the primary T-ALL sample (T-ALL 2) and the primary T-lymphoma samples (PT4-unclassified PTCL and SPT-1-Sezary Syndrome from bone marrow aspirate) after overnight co-culture using the indicated CD markers for gating. CD5CAR NK-92 cells also target and eliminate UCB-derived T-cells expressing CD5. All patient samples express a significant amount of CD5. (b) At a 5:1 E:T ratio, the CD5CAR NK-92 cells eliminate virtually all CD5 ${ }^{+}$populations derived from primary patient samples. (c) CD5CAR NK-92 cells target and lyse $\mathrm{CD} 5^{+} \mathrm{MCL}$ cell line JeKo and $\mathrm{CD} 5^{+}$primary mantle cell patient sample $\mathrm{L3}-\mathrm{G}$. Co-cultures were carried out at the indicated E:T ratios. (d) Absolute cell counts of CD5CAR NK-92 cells and vector control NK-92 cells against primary PTCL, T-ALL, mantle cell and normal UCB T-cells. Control and CD5CAR treatments are delineated in red and blue, respectively, with effector and target cells counted after culture. 
CD5CAR NK-92 specifically lyses CD5+ Patient Sample T-ALL 2 populations
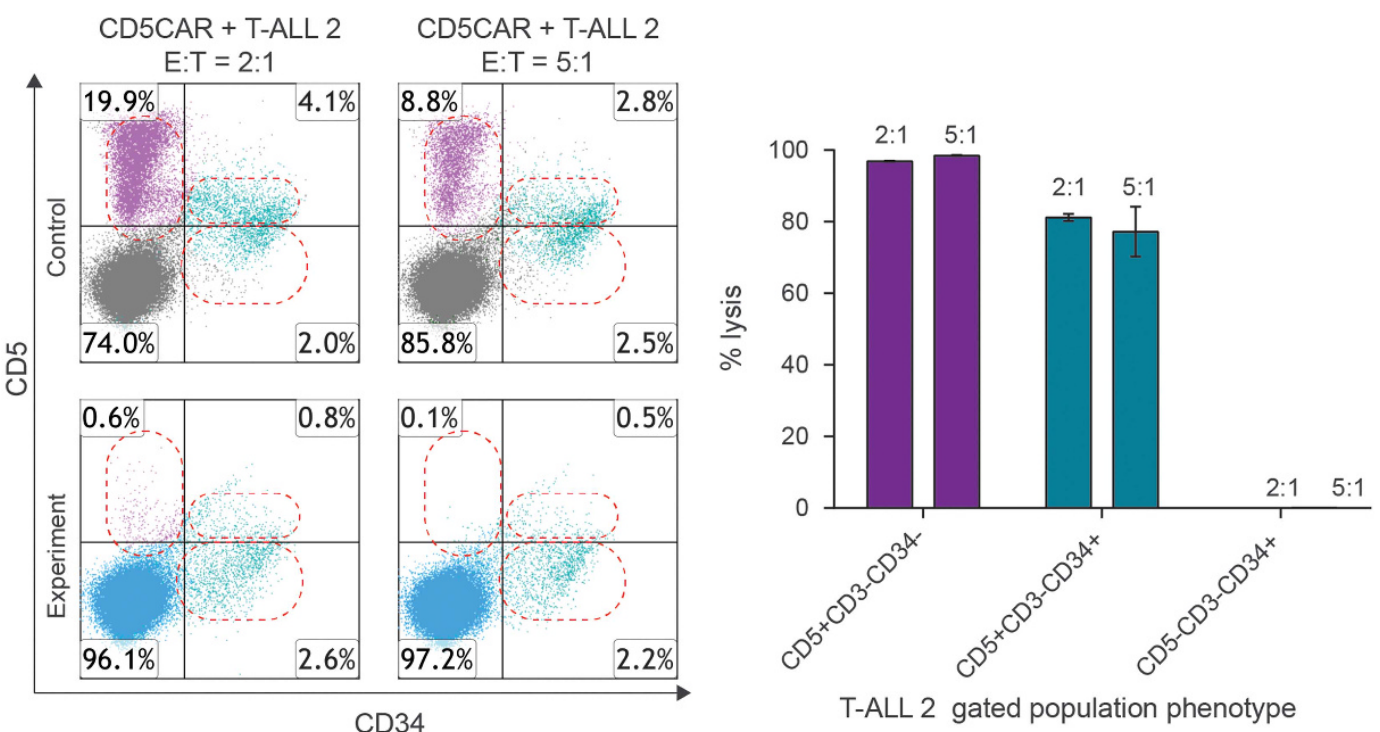

T-ALL 2 gated population phenotype

b CD5CAR NK-92 specifically lyses CD5+ Patient Sample L3-G populations
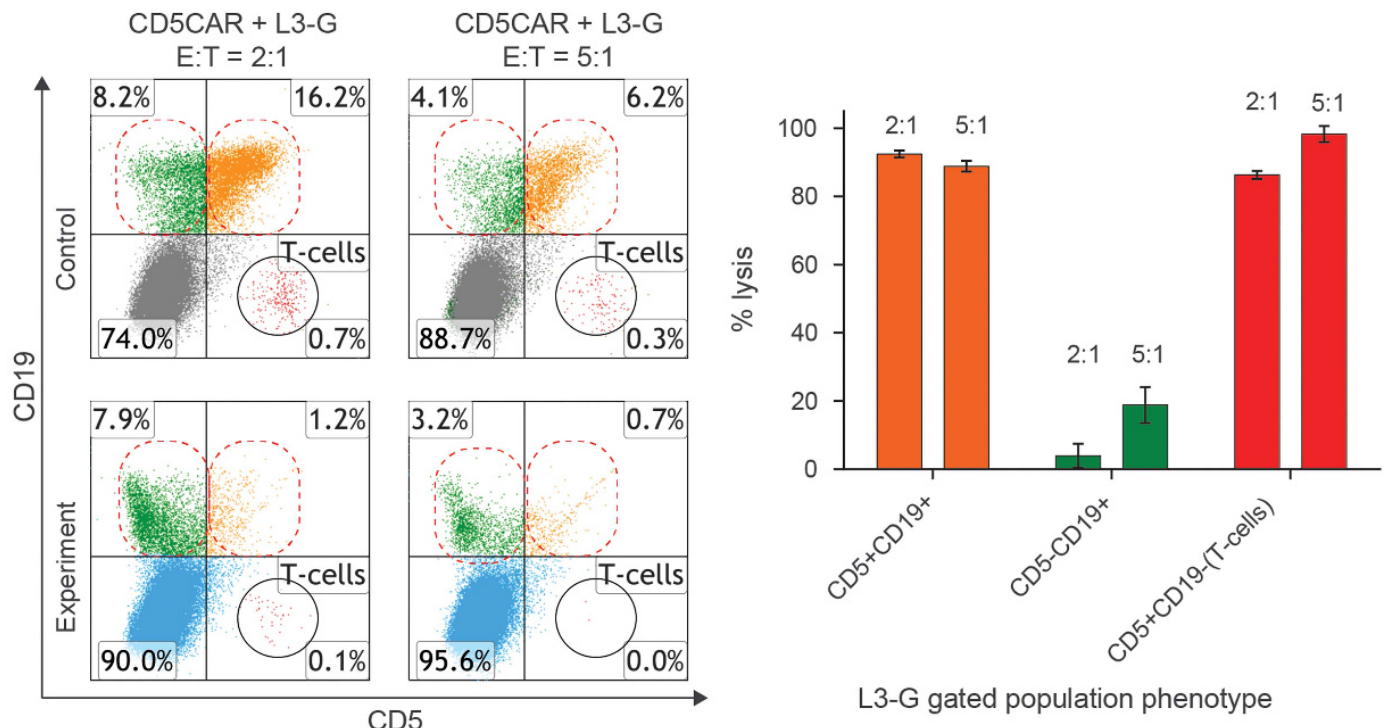

L3-G gated population phenotype

Figure 4. CD5CAR NK-92 cells demonstrate $C D 5^{+}$specific lysis with few off-target effects. (a) CD5CAR NK-92 cells show specific lysis of CD5 ${ }^{+}$ T-ALL 2 populations. $\mathrm{CD}^{+} \mathrm{CD}^{-} \mathrm{CD} 34^{-}$(purple, upper left quadrant) and $\mathrm{CD} 5^{+} \mathrm{CD} 3^{-} \mathrm{CD} 34^{+}$(teal, upper right quadrant) populations are targeted and lysed by CD5CAR NK-92. CD5 ${ }^{-}$CD3 ${ }^{-}$CD34 $4^{+}$cells (teal, lower right quadrant) remain unaffected. (b) CD5CAR NK-92 cells show specific lysis of $\mathrm{CD}^{+}$(B-cell MCL) populations. $\mathrm{CD}^{+} \mathrm{CD} 19^{+}$(orange, upper right quadrant) and $\mathrm{CD} 5^{+} \mathrm{CD} 19^{-}$(red, bottom right quadrant) populations are lysed by CD5CAR NK-92. $\mathrm{CD}^{-} \mathrm{CD} 19^{+}$cells (green, upper left quadrant) remain largely unaffected.

short-lived after tumorlysis. Therefore, in order to investigate potential dose-dependent relationships, we diluted the E:T ratios to $0.25: 1$ (25000 effector cells to 100000 target cells) for co-cultures with a variety of $\mathrm{CD}^{+}$cell types, both malignant and non-malignant: normal human T-cells; PT4 lymphoma cells; T-ALL 2 cells; and CCRF-CEM cells. We observed similar dosedependency trends for all treatments, suggesting that CD5CAR NK-92 cells are versatile and possess potent and consistent cytotoxicity mechanisms against all types of $\mathrm{CD}^{+}$cell types (Figures 5a-c and Supplementary Figure 5). In summary, these studies show that CD5CAR NK-92 cells exhibited profound and specific anti-tumor activity in leukemic cell lines and patient leukemic samples for T-ALL, PTCLs and B-cell lymphomas expressing CD5 (Figure 5d) with no effect on CD5-negative controls such as KARPAS.

CD5CAR NK cells demonstrate potent anti-leukemic activity in vivo Two animal studies were employed to determine the in vivo antitumor activity of CD5CAR NK-92 cells. Overall, CD5CAR NK-92 cells demonstrated robust control and suppression of Jurkat tumor growth in xenogeneic mouse models. Sublethally irradiated NSG mice were intravenously injected with $1.0 \times 10^{6}$ firefly luciferaseexpressing Jurkat cells to induce visible tumor formation through IVIS imaging by day 3 and onwards (Figures $6 a$ and b). CD5CAR NK-92 cells were then injected on day 4 based on a rationale for maximum efficacy, by administering a one-course dose of $15 \times 10^{6}$ 
a
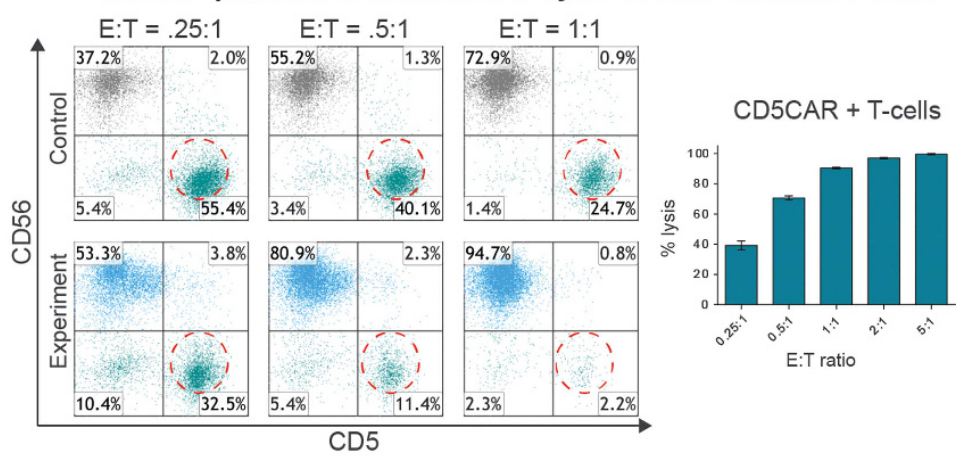

b

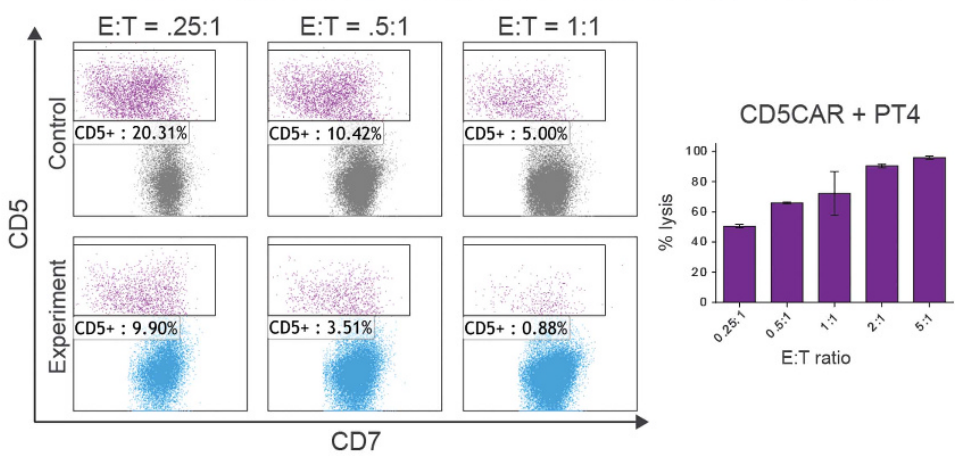

C

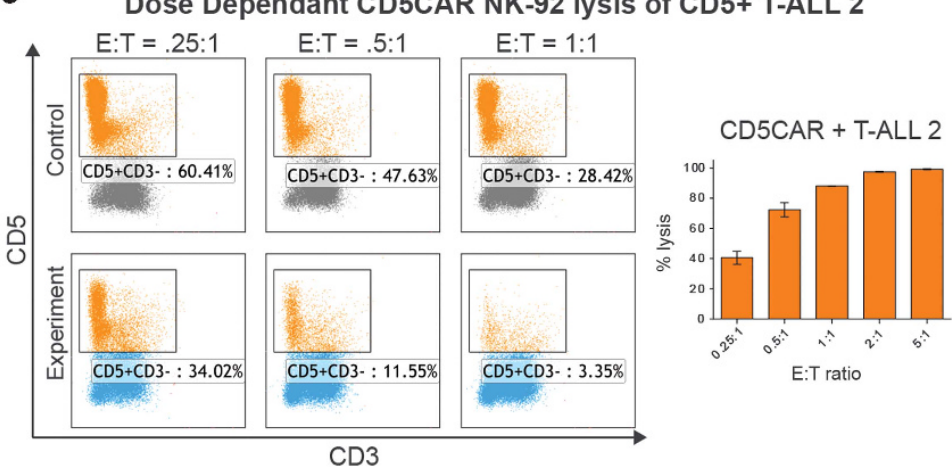

d Composite Comparison of CD5CAR NK-92 Lysis of $\mathrm{CD}^{+}$Cells at E:T Ratios of 2:1 and 5:1

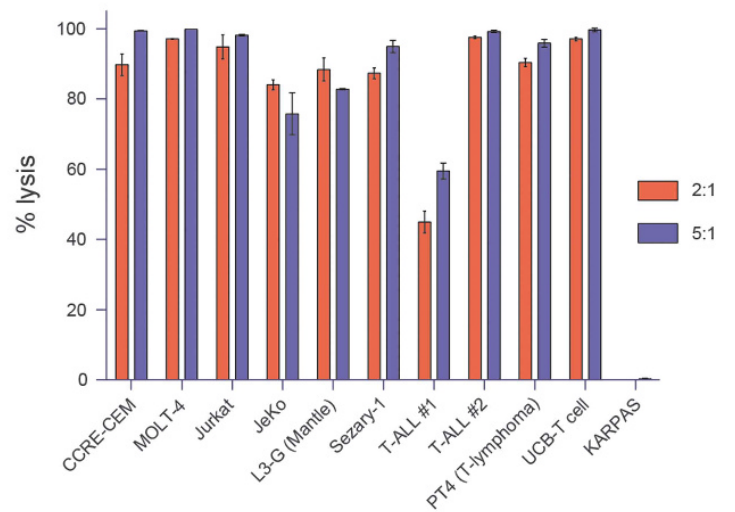

Figure 5. CD5CAR NK-92 cells exhibit dose-dependent target cell lysis. (a) CD5CAR NK-92 cells show dose-dependent lysis of normal primary patient T-cells as the ratio is increased from a lowered E:T ratio of 0.25:1 to standard E:T ratios of 5:1 and reaching saturation. (b) CD5CAR NK-92 cells lyse malignant CD5 ${ }^{+}$PT4 patient cells in a dose-dependent manner as the E:T ratio is increased, reaching lysis saturation at 5:1. (c) CD5CAR NK-92 cells lyse T-ALL 2 CD5 $^{+}$patient sample cells in a dose-dependent manner as the E:T ratio is increased, reaching saturation at 5:1 with significant activity observed for dosages as low as 0.25:1. (d) The CD5CAR NK-92 summary panel of CAR activity in lysing T-ALL cell lines and primary human samples. All T-ALL and primary samples expressing CD5 demonstrate targeting and lysis by CD5CAR NK-92 cells, with $8 / 10 \mathrm{CD}^{+}$samples showing a percentage lysis of $>80 \%$ for both $\mathrm{E}: \mathrm{T}$ ratios. Percent lysis values were determined using the total $\mathrm{CD} 5^{+}$ populations after gating for viability against the vector control NK-92 treatment.

CAR or control NK-92 cells during the NK-92 life expectancy of around 10 days for mouse model 1 (MM1) and $10 \times 10^{6}$ CAR or control NK-92 cells during the 10 day window for mouse model 2 (MM2).

To observe NK-92 mediated tumor control and quantitate tumor burden, we measured the average light intensity measured in photons per second for the CD5CAR NK-92 injected mice versus that of the vector control treatment (Figures $6 a$ and b). On days 6 and 11, mice were injected subcutaneously with RediJect D-Luciferin and subjected to IVIS imaging to measure tumor burden (Figures $6 a$ and b). By day 11, this led to a $59 \%$ reduction in tumor burden for MM1 and an $86 \%$ reduction for MM2, with increased control and maintenance of tumor burden by day 14 for both mouse models, the end point of the projected NK-92 lifespan. For MM1, on day 11, two mice died 30 minutes after NK-92 cell injection, most likely due to stroke from injection procedure and NK-92 cell aggregation. ${ }^{10}$ Similarly, on day 10, one mouse died as a result of injection procedure for MM2. Therefore, these mice were excluded from the survival curve and statistics pool for each model. To investigate the maintenance of tumor control, two additional low dose injections totaling $5 \times 10^{6}$ NK-92 cells were conducted through day 19 for MM1 and day 20 for $M M 2$. This resulted in continued suppression of tumor growth and reduction of tumor burden by $>75 \%$ and $>90 \%$ for $\mathrm{MM} 1$ and MM2, respectively (Figure $6 a$ and b). Consistent with earlier injection courses correlated with tumor reduction numbers, tumor 
Day 6

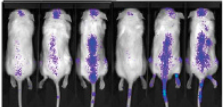

Day 11

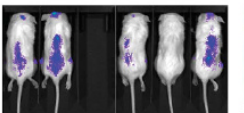

Day 15

Day 21

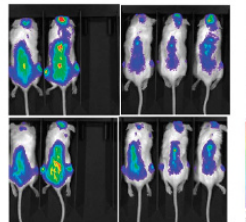

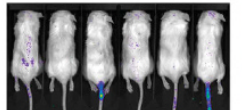
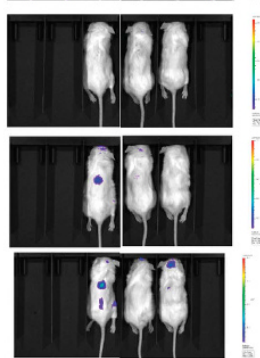

$1.0 \times 10 \mathrm{e} 5$

\section{$1.0 \times 10 \mathrm{e} 4$}

$5.1 \times 10 \mathrm{e} 5$

$5.1 \times 10 \mathrm{e} 4$

$1.0 \times 10 \mathrm{e} 6$

$1.0 \times 10 \mathrm{e} 5$

$2.0 \times 10 \mathrm{e} 7$

$2.0 \times 10 \mathrm{e} 6$
CD5CAR NK-92 cells control

$\mathrm{CD} 5^{+}$Jurkat tumor growth

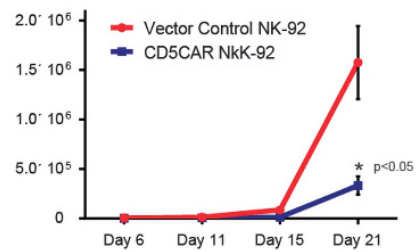

CDCAR NK-92 treatment reduces tumor burden

$\square$ Vector Control NK-92 $\square$ CD5CAR NK-92

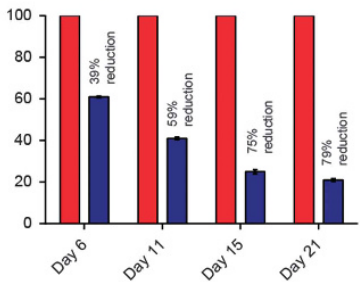

b

IVIS Imaging of Jurkat-Luc+ injected mouse model \#2

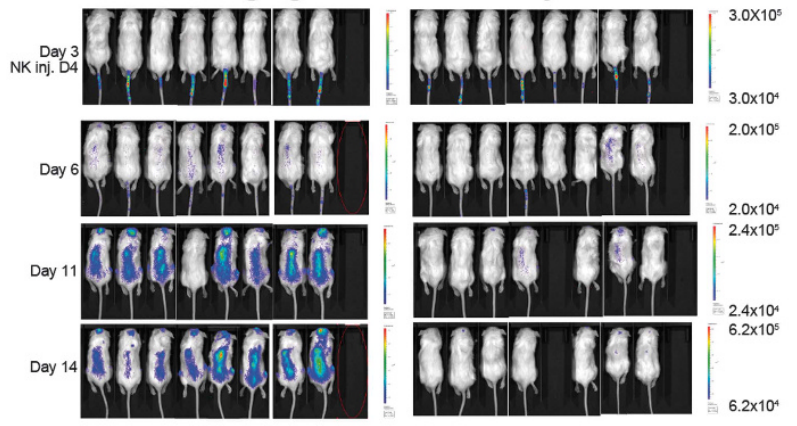

CD5CAR NK-92 cells control CD5 ${ }^{+}$Jurkat tumor growth

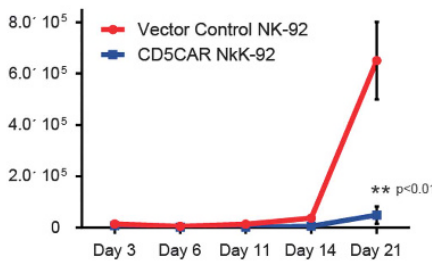

CD5CAR NK-92 treatment reduces tumor burden $\square$ Vector Control NK-92 - CD5CAR NK-92

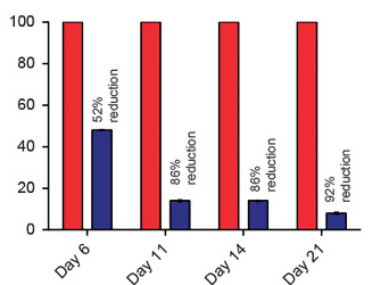

C

CD5CAR NK-92 effect on mouse survival

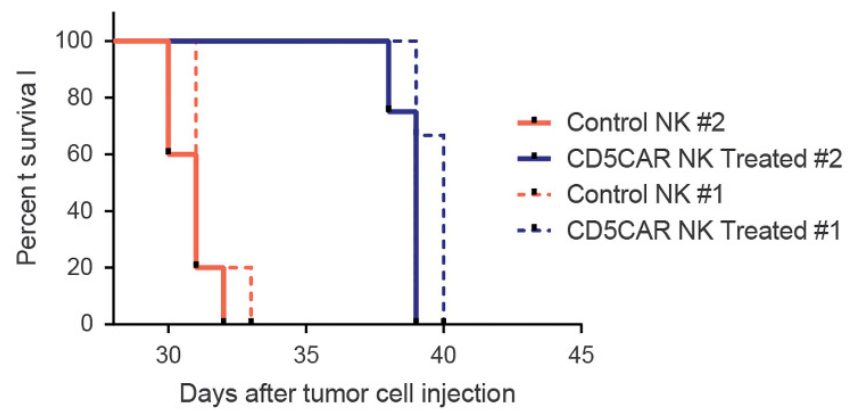

p-value $<0.01$ for both treatment groups against their respective controls

Figure 6. CD5CAR NK-92 cells demonstrate potent in vivo activity. (a) NSG mice were sublethally irradiated and, after 24 h, intravenously injected with $1 \times 10^{6}$ luciferase-expressing Jurkat cells (Day 1) to induce measurable tumor formation. Over the course of the NK-92 cell lifespan, mice were injected with a one course dose consisting of $15 \times 10^{6}$ control NK-92 or CD5CAR NK-92 cells concluding by day 11 . On day 11, 2 mice died within 30 min of injection process likely due to stroke and NK-92 cell aggregation during procedure. On days 6,11 , 15 and 21, mice were injected subcutaneously with RediJect D-Luciferin and subjected to IVIS imaging. Subsequent maintenance dose consisting of $5 \times 10^{6}$ cells was administered through day 20 . Control of the Jurkat-Luc ${ }^{+}$tumor growth was quantified via luciferin signal (average light intensity) and plotted as a line graph. Tumor reduction is normalized against the vector control NK cell treatment and expressed in bar graphs showing \% reduction. (b) A second group of NSG mice were injected with Jurkat-Luc ${ }^{+}$cells and treated with CD5CAR NK-92 cells with a onecourse dose consisting of $10 \times 10^{6}$ cells over the course of the NK-92 cell lifespan. On day 10, one mouse died as a result of injection procedure. RediJect luciferin was injected on days 3, 6, 11 and 14. Subsequent maintenance dose consisting of $5 \times 10^{6}$ cells was injected through day 19. Day 3 data was obtained to show tumor after Day 1 Jurkat-Luc ${ }^{+}$injection. (c) CD5CAR NK-92 treated mice survive significantly longer than control in both Jurkat-Luc ${ }^{+}$mouse models. Kaplan-Meier survival curve for CD5CAR NK-92 treated mice compared to vector control treated mice (Dotted lines represent Jurkat-Luc ${ }^{+}$group 1, solid lines represent group 2). Log-rank (Mantel-Cox) test $P$-values as shown.

control remained only consistent and did not result in complete eradication of engrafted Jurkat cells (Figures $6 a$ and b). Future experiments could be conducted to determine a more optimal dose related to efficiency and infused cell numbers.

In both xenogeneic mouse models, CD5CAR NK-92 cells robustly reduced tumor burden, controlled tumor growth, and significantly prolonged survival in the Jurkat-injected NSG mice when compared to control NK-92 treatment (Figures 6a-c). Paired $t$-test analysis of the tumor burden revealed highly significant differences $(P<0.01)$ between each treatment group and their respective controls (Figures $6 \mathrm{a}$ and b). Similar significant improved survival rates were observed for CD5CAR treated mice in both models (Figure 6c). CD5CAR NK-92 cells failed to completely eradicate established Jurkat tumor cells and leukemic cells did 
ultimately re-emerge from murine reservoirs likely inaccessible to NK-92 cell infiltration. CD5CAR NK-92 cells may also have been impacted by the lack of murine suitability for optimal human NK cell homing and persistence as we were unable to detect CD5CAR NK-92 cells in blood, bone marrow and spleen beyond day 30 (data not shown).

\section{DISCUSSION}

T-cell leukemia and lymphomas are aggressive cancers with very few curative options, limited treatment standards, and poor prognoses. In our studies, we show that a CD5CAR-redirected human NK cell line, NK-92, could be posited as a complement therapy to CD5CAR transduced T-cells ${ }^{29}$ with the potential benefits of transient NK-cell specific activity, and a safety profile in line with most CAR therapy targets. ${ }^{5,14,30}$ We found that CD5CAR NK-92 cells possessed potent and specific target directed anti-tumor effects in vitro. Furthermore, CD5CAR NK-92 cells were able to undergo stable expansion ex vivo and possessed efficacious and targeted cell-lytic properties to effectively control tumor growth in xenograft models of T-ALL.

CD5 is strongly expressed on the surface of normal and malignant T-cells prompting concerns that CARs targeting T-cell malignancies will incur T-cell aplasia similar to the B-cell aplasia observed for CD19CAR clinical trials. However, anti-CD5monoclonal antibody clinical trials have shown some efficacy in combating $\mathrm{CD}^{+}$malignancies and self-reactive T-cells without major irreversible toxicity. A lack of persistency and sustained effect by the murine antibodies suggest at the need for more targeted and potent therapy. ${ }^{24,31} \mathrm{~A}$ recent report ${ }^{29}$ showed that the level of cytotoxicity against normal T cells by CD5 CAR T cells was limited, but this was not the case for our CD5CAR NK cells. CD5CAR NK cells lysed normal T-cells as efficiently as CD5 ${ }^{+}$ malignant cells (Figure 3). We also generated CD5CAR T-cells using a CD28 co-stimulatory domain (unpublished data) and tested their ability to lyse normal $T$ cells with the result that our CD5CAR T-cells efficiently eliminated normal T-cells comparable to CD5CAR NK cells. The different levels of cytotoxicity against normal T-cells by our CD5CAR and the previous report ${ }^{29}$ may be due to the choice of the anti-CD5 scFv modulating potentially different mechanisms requiring further exploration as well as optimizing different effector : target cell ratios.

CD5CAR NK-92 cells showed profound efficacy, specificity, and potency in anti-tumor applications in vitro. CD5CAR NK-92 cells were able to lyse malignant target cell lines at $>95 \%$ efficacy for all co-cultures with Jurkat, CCRF-CEM, and MOLT-4 cells. In addition, CD5CAR NK-92 cells were able to produce potent and specific anti-tumor effects on primary malignant T-cell disease samples, with virtual elimination of target tumor populations without effects on CD5-negative population subsets (Figure 4). In addition, CD5CAR NK cells were able to effectively target $\mathrm{MCL}$, an aggressive $\mathrm{CD}^{+}$B-cell lymphoma comprising of $3-10 \%$ of B-cell lymphomas (Figure 3c). MCLs (which usually expresses CD5) severely lack reliable curative treatments and frequently relapse with a characteristic inexorable pattern of disease progression. Our results with CD5CAR NK cells against mantle cell samples suggest an avenue of potential application for this disease.

The in vivo efficacy of CD5CAR NK-92 cells are comparable to the CD5CAR T-cells in suppressing and eliminating engrafted tumor cells in xenograft mouse models and can potentially be a similar predictor for therapeutic efficacy in clinic. ${ }^{29}$ We established two xenograft models of T-ALL using luciferase-expressing Jurkat cells, and demonstrate that within the window of NK cell life expectancy, tumor burden was substantially reduced by over $50 \%$ in mouse model 1 and over $80 \%$ in mouse model 2 by Day 11 . Subsequent injections beyond the initial course served as a maintenance therapy for tumor suppression with the need for multiple injections an indicator for NK cell transiency. Consistent with this notion, shortly after CD5CAR NK-92 injections were halted, tumor re-emerged from both mouse models suggesting at the inability of injected NK-92 cells to completely eradicate Jurkat tumor growth and the limited ability of NK-92 cells to persist in the murine body without supportive cytokines and environment. Such tumor re-emergence from various murine reservoirs have been documented in various xenograft models $s^{4,29,32}$ before. To test the idea of NK transiency, we conducted injections of up to $6 \times 10^{7}$ CD5CAR NK-92 cells into mice and could not detect NK-92 cells in PB, spleen or bone marrow after 2 weeks (data not shown). NK cell therapy may thus need to include multiple dose injections as opposed to single dose CAR T injections for the same persistency observations. However, in clinic, NK cells could be allogenic and could be stabilized in a human microenvironment.

Unlike T-cells, NK-based therapies will have to balance the persistency of NK cells with tumor-lytic efficacy. Such treatments may be more suitable as quick-hitting transient treatments for the depletion of target tumor cells or self-reactive T-cells in preparation for transplantation. The potential disadvantages of using NK cells in CAR therapy include a lack of persistency and difficulty of high efficiency CAR transduction that may reduce long-term efficacy (which may be overcome by multi-administrations of CD5CAR NK cells as suggested by our dosage experiments). While the use of CD5CAR primary NK cells is important for clinical outlook, the cell culture issues of autologous primary NK cells are well documented ${ }^{11}$ and, in particular, are difficult to transduce with high efficiency. However, given the nature of NK cells, such therapy may alternatively serve to reduce the effect of cytokine storm or other inflammatory reactions elicited through usual CART treatment. ${ }^{33}$

Due to these properties of NK cells, NK therapy has potential in transient depletion of tumor cells as a result of inherently shorter lifespans and persistency mechanisms. We postulate that NK CAR therapy may be used as an 'off-the-shelf', transient cell therapy. Dosage experiments with CD5CAR show a linear association between CD5CAR NK-92 quantities vs target cell populations suggesting that mean numbers of NK cell infusion may relate to tumor-lytic efficacy, which can be optimized for clinical outcomes. With a 'hit and run' approach, NK cells can be used as a standalone treatment or a bridge therapy, that is, for minimal residual disease in preparation for bone marrow transplant. Because NK cells are exhausted shortly thereafter, persistency and cytokine issues with T-cells are avoided. The off-target toxicity of T-cell depletion, when the CD5CAR is applied, may be expected. Therefore, developing strategies to identify therapeutic windows, which patients can be tolerant, is important for CD5CAR therapy. A safety switch in CD5CAR is preferred and that should increase its safety although NK CAR therapy could be considered a transient treatment.

In conclusion, we have shown that CD5CAR transduced NK-92 cells have potent efficacy effects in vitro and in vivo that show significantly improved survival outcomes for CD5CAR treated mice. Our study projects that CD5CAR NK cells could be used alone in a short-term window or act as a bridge therapy for standard care procedures.

\section{CONFLICT OF INTEREST}

Yupo Ma is a co-founder of iCell Gene Therapeutics, LLC.

\section{ACKNOWLEDGEMENTS}

We thank Todd Rueb and Rebecca Connor for valuable technical assistance with cell sorting and flow cytometry set-up, and Yelena Altshuller for assistance with molecular cloning. 


\section{AUTHOR CONTRIBUTIONS}

KHC designed and performed the experiments, interpreted data and wrote the manuscript; MW designed and performed the experiments, interpreted data and manuscript revision; KGP performed the experiments; HL performed experiments; KL performed experiments; AJ assisted with review and revisions; AEF wrote the manuscript; XS performed experiments; HS provided clinical data accrual and suggestions; MG provided clinical data accrual and data review; FL provided clinical data accrual; LS provided clinical data accrual and sample acquisition; LL provided manuscript support, $\mathrm{XJ}$ provided manuscript suggestions and support; YM directed experimental overview, interpreted data and revised manuscript.

\section{REFERENCES}

1 Foss FM, Zinzani PL, Vose JM, Gascoyne RD, Rosen ST, Tobinai K. Peripheral T-cell lymphoma. Blood 2011; 117: 6756-6767.

2 Firor $A E$, Jares $A$, Ma Y. From humble beginnings to success in the clinic: chimeric antigen receptor-modified T-cells and implications for immunotherapy. Exp Biol Med (Maywood) 2015; 240: 1087-1098.

3 Brentjens RJ, Davila ML, Riviere I, Park J, Wang X, Cowell LG et al. CD19-targeted $T$ cells rapidly induce molecular remissions in adults with chemotherapyrefractory acute lymphoblastic leukemia. Sci Transl Med 2013; 5: 177ra138.

4 Brentjens RJ, Santos E, Nikhamin Y, Yeh R, Matsushita M, La Perle K et al. Genetically targeted $\mathrm{T}$ cells eradicate systemic acute lymphoblastic leukemia xenografts. Clin Cancer Res 2007; 13(18 Pt 1): 5426-5435.

5 Arai S, Meagher R, Swearingen M, Myint H, Rich E, Martinson J et al. Infusion of the allogeneic cell line NK-92 in patients with advanced renal cell cancer or melanoma: a phase I trial. Cytotherapy 2008; 10: 625-632.

6 Pinz K, Liu H, Golightly M, Jares A, Lan F, Zieve GW et al. Preclinical targeting of human T-cell malignancies using CD4-specific chimeric antigen receptor (CAR)engineered T cells. Leukemia 2016; 30: 701-707.

7 Godfrey J, Benson DM Jr.. The role of natural killer cells in immunity against multiple myeloma. Leuk Lymphoma 2012; 53: 1666-1676.

8 Romanski A, Uherek C, Bug G, Seifried E, Klingemann H, Wels WS et al. CD19-CAR engineered NK-92 cells are sufficient to overcome NK cell resistance in B-cell malignancies. J Cell Mol Med 2016; 20: 1287-1294.

9 Chu J, Deng Y, Benson DM, He S, Hughes T, Zhang J et al. CS1-specific chimeric antigen receptor (CAR)-engineered natural killer cells enhance in vitro and in vivo antitumor activity against human multiple myeloma. Leukemia 2014; $\mathbf{2 8}$ : 917-927.

10 Chen KH, Wada M, Firor AE, Pinz KG, Jares A, Liu H et al. Novel anti-CD3 chimeric antigen receptor targeting of aggressive T cell malignancies. Oncotarget 2016; 7: 56219-56232.

11 Glienke W, Esser R, Priesner C, Suerth JD, Schambach A, Wels WS et al. Advantages and applications of CAR-expressing natural killer cells. Front Pharmacol 2015; 6: 21.

12 Klingemann $\mathrm{H}$, Boissel L, Toneguzzo F. Natural killer cells for immunotherapy advantages of the NK-92 cell line over blood NK cells. Front Immunol 2016; 7: 91.

13 Suck G, Odendahl M, Nowakowska P, Seidl C, Wels WS, Klingemann HG et al. NK-92: an 'off-the-shelf therapeutic' for adoptive natural killer cell-based cancer immunotherapy. Cancer Immunol Immunother 2016; 65: 485-492.

14 Tonn T, Schwabe D, Klingemann HG, Becker S, Esser R, Koehl U et al. Treatment of patients with advanced cancer with the natural killer cell line NK-92. Cytotherapy 2013; 15: 1563-1570.

15 Hoyos V, Savoldo B, Quintarelli C, Mahendravada A, Zhang M, Vera J et al. Engineering CD19-specific T lymphocytes with interleukin-15 and a suicide gene to enhance their anti-lymphoma/leukemia effects and safety. Leukemia 2010; 24: 1160-1170.

16 Wu C, Hong SG, Winkler T, Spencer DM, Jares A, Ichwan B et al. Development of an inducible caspase- 9 safety switch for pluripotent stem cell-based therapies. Mol Ther Methods Clin Dev 2014; 1: 14053.
17 Schonfeld K, Sahm C, Zhang C, Naundorf S, Brendel C, Odendahl M et al. Selective inhibition of tumor growth by clonal NK cells expressing an ErbB2/HER2-specific chimeric antigen receptor. Mol Ther 2015; 23: 330-338.

18 Zhang C, Burger MC, Jennewein L, Genssler S, Schonfeld K, Zeiner P et al. ErbB2/HER2-specific NK cells for targeted therapy of glioblastoma. J Nat/ Cancer Inst 2016; 108: djv375.

19 Boissel L, Betancur M, Wels WS, Tuncer H, Klingemann H. Transfection with mRNA for CD19 specific chimeric antigen receptor restores NK cell mediated killing of CLL cells. Leuk Res 2009; 33: 1255-1259.

20 Boissel L, Betancur-Boissel M, Lu W, Krause DS, Van Etten RA, Wels WS et al. Retargeting NK-92 cells by means of CD19- and CD20-specific chimeric antigen receptors compares favorably with antibody-dependent cellular cytotoxicity. Oncoimmunology 2013; 2: e26527.

21 Jiang $H$, Zhang W, Shang P, Zhang H, Fu W, Ye F et al. Transfection of chimeric anti-CD138 gene enhances natural killer cell activation and killing of multiple myeloma cells. Mol Oncol 2014; 8: 297-310.

22 Pui CH, Behm FG, Crist WM. Clinical and biologic relevance of immunologic marker studies in childhood acute lymphoblastic leukemia. Blood 1993; 82: 343-362.

23 Campana D, van Dongen JJ, Mehta A, Coustan-Smith E, Wolvers-Tettero IL, Ganeshaguru K et al. Stages of T-cell receptor protein expression in T-cell acute lymphoblastic leukemia. Blood 1991; 77: 1546-1554.

24 Strand V, Lipsky PE, Cannon GW, Calabrese LH, Wiesenhutter C, Cohen SB et al. Effects of administration of an anti-CD5 plus immunoconjugate in rheumatoid arthritis. Results of two phase II studies. The CD5 plus rheumatoid arthritis investigators group. Arthritis Rheum 1993; 36: 620-630.

25 Siena S, Bregni M, Formosa A, Brando B, Marenco P, Lappi DA et al. Immunotoxinmediated inhibition of chronic lymphocytic leukemia cell proliferation in humans. Cancer Res 1989; 49: 3328-3332.

26 Wacholtz MC, Lipsky PE. Treatment of lupus nephritis with CD5 PLUS, an immunoconjugate of an anti-CD5 monoclonal antibody and ricin A chain. Arthritis Rheum 1992; 35: 837-839.

27 Fishwild DM, Strand V. Administration of an anti-CD5 immunoconjugate to patients with rheumatoid arthritis: effect on peripheral blood mononuclear cells and in vitro immune function. J Rheumatol 1994; 21: 596-604.

28 Kochenderfer JN, Rosenberg SA. Treating B-cell cancer with T cells expressing anti-CD19 chimeric antigen receptors. Nat Rev Clin Oncol 2013; 10: 267-276.

29 Mamonkin M, Rouce RH, Tashiro H, Brenner MK. A T-cell-directed chimeric antigen receptor for the selective treatment of T-cell malignancies. Blood 2015; 126: 983-992.

30 Tonn T, Becker S, Esser R, Schwabe D, Seifried E. Cellular immunotherapy of malignancies using the clonal natural killer cell line NK-92. J Hematother Stem Cell Res 2001; 10: 535-544.

31 Antin JH, Bierer BE, Smith BR, Ferrara J, Guinan EC, Sieff C et al. Selective depletion of bone marrow $\mathrm{T}$ lymphocytes with anti-CD5 monoclonal antibodies: effective prophylaxis for graft-versus-host disease in patients with hematologic malignancies. Blood 1991; 78: 2139-2149.

32 Barrett DM, Liu X, Jiang S, June CH, Grupp SA, Zhao Y. Regimen-specific effects of RNA-modified chimeric antigen receptor $T$ cells in mice with advanced leukemia. Hum Gene Ther 2013; 24: 717-727.

33 Klingemann $\mathrm{H}$. Are natural killer cells superior CAR drivers? Oncoimmunology 2014; 3: e28147.

(c) (i) $(\$)$ This work is licensed under a Creative Commons Attributioncc NonCommercial-NoDerivs 4.0 International License. The images or other third party material in this article are included in the article's Creative Commons license, unless indicated otherwise in the credit line; if the material is not included under the Creative Commons license, users will need to obtain permission from the license holder to reproduce the material. To view a copy of this license, visit http:// creativecommons.org/licenses/by-nc-nd/4.0/

(c) The Author(s) 2017

Supplementary Information accompanies this paper on the Leukemia website (http://www.nature.com/leu) 